\title{
Graphite-like dynamical behaviour of graphite oxide
}

\author{
Amul Shinde, Christoph Testud, Katrin Adamczyk, Matthias Ruppert, and Nils Huse* \\ Institute for Nanostructure and Solid State Physics and SFB 925, Department of Physics, \\ University of Hamburg, Center for Free Electron Laser Science, Luruper Chaussee 149, \\ 22761 Hamburg, Germany
}

\begin{abstract}
We report two-colour pump-probe spectroscopy of coupled structural and electronic dynamics of graphite oxide probed with $6-\mu \mathrm{m}$ and THz pulses upon femtosecond IR excitation.
\end{abstract}

\section{Introduction}

Since the discovery of graphene's unique electronic structure with linear dispersion of Dirac electrons graphite oxide (GO) has gained attention due to its potential in mass production of graphene. However, it is increasingly apparent that GO is an interesting material of its own with various applications from plastic electronics, optical materials and catalysis to biosensors. Its heterogeneous chemical and electronic structures engender from a variety of oxygen functional groups which have been identified as hydroxyl $(\mathrm{OH})$, epoxy $(-\mathrm{O}-)$, carbonyl $(\mathrm{C}=\mathrm{O})$, and carboxylic $(-\mathrm{COOH})$ groups alongside five- and six-memberedring lactols. These groups are attached to the two-dimensional graphene layer, thereby distorting the planar geometry of the graphene sheets [1]. The functionalization of graphene thus leads to non-stoichiometric macromolecules with a complicated structure. The particular distribution of these functional groups will determine domain structure and local band gaps as well as carrier dynamics and energy transfer mechanisms. Due to the oxygencontaining functional groups GO is highly hygroscopic. As a consequence, water is trapped within the $\sim 1 \mathrm{~nm}$ spacing between individual graphene oxide sheets [2], leading to additional energy relaxation channels.

We report on two-colour pump-probe spectroscopy to study vibrational/phonon and carrier dynamics after excitation of the $\sim 14 \mu \mathrm{m}$ thick self-supporting GO membrane with femtosecond pulses from the UV to the mid-infrared (MIR) and probed the material response in the MIR and THz region. Probing in the MIR spectral region gives access to vibrational (phonon) dynamics, vibrational (phonon-phonon) coupling and vibronic (electron-phonon) coupling between electronic and nuclear degrees of freedom. The THz spectral region complements the insight into the materials response to yield information on charge carrier dynamics as they exhibit a distinct optically active response in the MIR and $\mathrm{THz}$ frequency range.

"Corresponding author: nils.huse@uni-hamburg.de 


\section{Experimental and results}

Ultrafast responses of GO in the MIR region upon $2-\mu \mathrm{m}(620 \mathrm{meV})$ excitation are shown in Fig. 1B with static absorption (top) and time-resolved differential absorption spectra (bottom). The MIR spectral region between 1500 and $1900 \mathrm{~cm}^{-1}$ is resonant with the inplane stretching modes (phonons) of the $\mathrm{sp}^{2}$-hybridized $\mathrm{C}=\mathrm{C}$ and carbonyl $(\mathrm{C}=\mathrm{O})$ groups. Upon excitation at $2 \mu \mathrm{m}$ the transient MIR spectra show two distinct features superimposed on a broad transient background (characteristic of free charge carriers). These two spectral features and the electronic background appear within the experimental time resolution of $150 \mathrm{fs}$ and subsequently decay with very similar kinetics. The transient inverted band around $1570 \mathrm{~cm}^{-1}$ can be assigned to a reduced $\mathrm{C}=\mathrm{C}$ stretching response. The second inverted band around $1673 \mathrm{~cm}^{-1}$ can be assigned to the in-plane carbonyl stretching vibration of an $\alpha, \beta$-unsaturated carbonyl, the so called enone (Fig. 1A illustrated the enone functional group within a GO sheet).

A
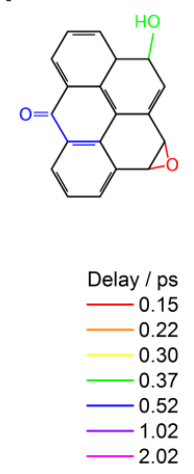
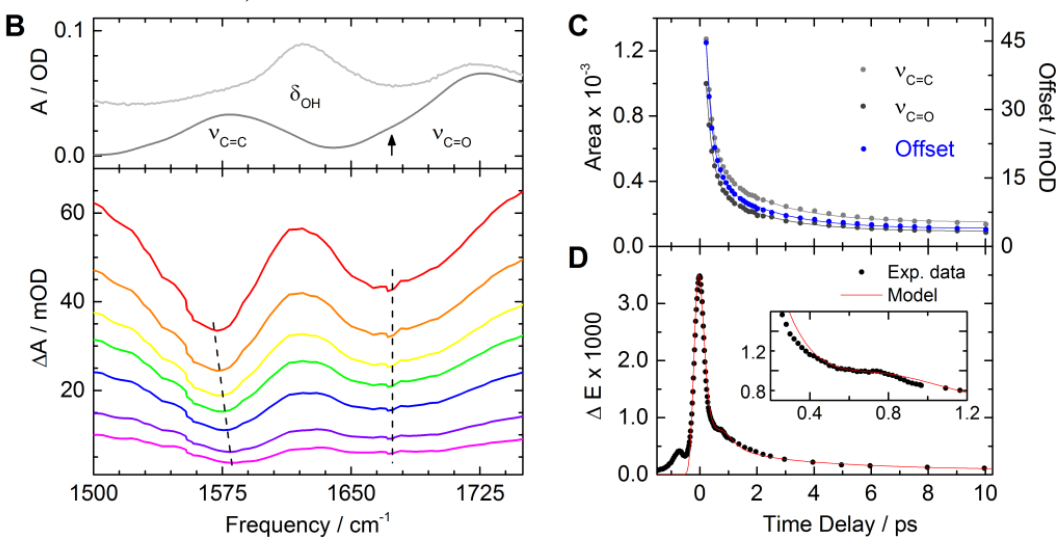

Fig. 1. A Schematic GO model with enone (blue), epoxy (red) and hydroxyl (green) groups. B Static and transient spectra of GO $(\Delta \mathrm{t}: 0.15 \mathrm{ps}$ to $2 \mathrm{ps})$. C Time traces of the $v_{\mathrm{C}=\mathrm{C}}, v_{\mathrm{C}=\mathrm{O}}$, and carrier response. D THz response of GO after $2 \mu \mathrm{m}$ excitation.

In order to quantitatively retrieve the decay time constants for each signal contribution a line-shape fitting procedure has been used to model the two transient bands with asymmetric Gaussians and the broad electronic background. The evolution of selected structural line-shape parameters and the electronic background (offset) are displayed in Fig. 1C. A modified two-temperature model with the shortest two time constants of $150 \mathrm{fs}$ and $600 \mathrm{fs}$ and an intermediate time constant of about $4 \mathrm{ps}$ can be fit to the area of both transient bands and the broad underlying signal independent of the pump wavelength. The frequency shift of the $C=C$ stretching vibration occurs with a time constant of about $600 \mathrm{fs}$. A small long-time component extends to hundreds of picoseconds.

The first time constant can be assigned to carrier-phonon scattering. For graphite photoexcited carriers transfer most of their energy to a set of strongly coupled optical phonons (SCOPs), including the $\mathrm{C}=\mathrm{C}$ ring stretching vibration (G-mode phonon) within less than $300 \mathrm{fs}$ [3] leading to a significant non-equilibrium phonon population. Subsequent phononphonon scattering proceeds with a time constant of $2.2 \mathrm{ps}$. The cooling process manifests itself in a relaxation of the G-phonon frequency shift of about $10 \mathrm{~cm}^{-1}$ upon carrier relaxation due to electron-phonon coupling increasing to that of the unexcited sample, reminiscent of dynamical behaviour in graphene and graphite. [3] Compared to graphite the 600 fs time constant points to a faster cooling process in GO. Possibly due to the higher vibrational density of states in GO compared to graphite in part provided by the oxygencontaining functional groups and intercalated water. The observation that the in-plane 
carbonyl stretching vibration shows the very same dynamics as the $\mathrm{C}=\mathrm{C}$ stretching vibration hints at strong coupling between these two modes. Indeed, a recent twodimensional infrared spectroscopic study of a series of enone-containing quinones reports significant vibrational coupling between these two modes [4].

GO studies employing Raman spectroscopy, scanning tunnelling microscopy and highresolution transmission electron microscopy report irregular coverage of oxygen-containing functional groups on the graphene sheets [1]. Ordered small $\mathrm{sp}^{2}$ carbon domains exist within the $\mathrm{sp}^{3} \mathrm{C}-\mathrm{O}$ matrix. Calculations have shown that such isolated molecular $\mathrm{sp}^{2}$ domains within the $\mathrm{sp}^{3}$ matrix can lead to confinement of $\pi$-electrons in GO [5]. The smaller these $\mathrm{sp}^{2}$ domains, the higher the local energy gap due to increased spatial confinement. Conversely, the large bandgap distribution manifesting in absorption from $\mathrm{meV}$ to $\mathrm{eV}$ reflect a large range of $\mathrm{sp}^{2}$ domain sizes in $\mathrm{GO}$ without a distinct band gap. Our experimental findings show that photo-generation of electron-hole pairs is feasible even at photon energies of $\sim 200 \mathrm{meV}$ and below. This implies the existence of $\mathrm{sp}^{2}$ domains substantially larger than $2 \mathrm{~nm}$ but other $\mathrm{sp}^{2}$ configurations such as different shapes and topologies and in-plane edge carbonyl groups present in GO should be considered as well.

The generation of charge carriers in GO induced by optical excitation at $2 \mu \mathrm{m}$ has also been probed as a change in the $\mathrm{THz}$ peak electric field. The resulting kinetic trace is depicted in Fig. 1D. Besides the carrier decay dynamics the $\mathrm{THz}$ probe pulses may also induce dynamics in the sample as well. For a correct description of the $\mathrm{THz}$ response, we use a third-order approach which includes finite dephasing times for the THz-induced polarization and proper convolution of response functions. By doing so, the elevation at positive times can be correctly modelled, also by using the time constants gained from IR probe measurements. The signals at negative time delays may be interpreted as perturbed free induction decay which would explain the discrepancy between pump pulse duration and the EOS response function on one hand and the apparently larger $\mathrm{THz}$ response of GO with recurrences reminiscent of the field envelope of the THz probe pulse on the other.

Interpretation of such kind of decay is under current discussion in the literature which leads us to mention that electron-electron and electron-phonon scattering play a key role in the decay mechanism during the first picoseconds [6] but the time constants for these are not clearly attributed in the literature, yet [7]. We suspect that the last decay step is via trapping of carriers in mid-gap states arising from numerous defects leading to relaxation timescales of tens of picoseconds. Small persistent signals on timescales of hundreds of picoseconds point to occupied defect states and diffusive carrier motion with increasingly scarce unoccupied trapping states.

\section{References}

1. K. P. Loh, Q. Bao, G. Eda, and M. Chhowalla, Nature Chemistry 2, 1015 (2010)

2. M. Acik, C. Mattevi, C. Gong, G. Lee, K. Cho, M. Chhowalla, and Y. J. Chabal, ACS Nano 4, 5861 (2010)

3. H. Yan, D. Song, K. F. Mak, I. Chatzakis, J. Maultzsch, and T. F. Heinz, Physical Review B 80, 121403(R) (2009)

4. J. D. Cyran, J. M. Nite, and A. T. Krummel, J. Phys. Chem. B 119, 8917 (2015)

5. G. Eda, Y.-Y. Lin, C. Mattevi, H. Yamaguchi, H.-A. Chen, I-S. Chen, C.-W. Chen, and M. Chhowalla, Adv. Mater. 22, 505 (2010)

6. P. A. George, J. Strait, J. Dawlaty, S. Shivaraman, M. Chandrashekhar, F. Rana and M. Spencer, Nano. Lett. 8, 4248 (2008)

7. Y. Yang, G. Kolesov, L. Kocia, and E. J. Heller, Nano Lett. 17, 6077 (2017) 